\title{
AVALIAÇÃO EXPERIMENTAL DA SENSIBILIDADE DO BIODIESEL B5 EM MOTORES DIESEL UTILIZADO EM FROTAS DE ÔNIBUS URBANO E INTERURBANO
}

\author{
M. F. Oliveira Filho ${ }^{1,2,3}$, L. C. da Silva ${ }^{1,3}$, M. A. de Abreu ${ }^{2}$, A. S. M. Galvão Filho ${ }^{4}$, A. C. M. Farias ${ }^{1,3}$, C. R. F. \\ Barbosa $^{1,3}$, J. T. N. Medeiros ${ }^{1,3}$ \\ ${ }^{1}$ Universidade Federal do Rio Grande do Norte, ${ }^{2}$ Instituto Federal do Rio Grande Norte Universidade - Campus \\ Natal Central, ${ }^{3}$ GET - Grupo de Estudos de Tribologia - Natal/RN e Transporte Cidade do Natal Ltda, Natal/ RN \\ manoel.fernandes@ifrn.edu.br, ledchagas@hotmail.com, marcel_1310@hotmail.com, salemaf@hotmail.com, \\ alinecmfarias@yahoo.com, cleiton@ufrnet.br, jtelesforo@yahoo.com
}

Artigo submetido em julho/2011 e aceito em maio/2012

\section{RESUMO}

O estudo objetiva investigar a consolidação do uso do biodiesel em motores de duas frotas de ônibus, uma urbana e uma interurbana. Entende-se serem necessários estudos para se observar tecnologicamente se o uso do biodiesel apresenta problemas quanto ao desgaste das peças diretamente envolvidas com seu uso e também observar as flutuações, para mais ou para menos, do consumo específico desse combustível. O diesel fóssil - tipo D e Biodiesel B5 foram analisados analiticamente, investigando-se também o teor de enxofre presente. Duas frotas de ônibus urbana e interurbana possuem 41 e 13 veículos, respectivamente. Foram analisadas as planilhas de cada veículo no período de três anos (2008 a 2010) e entrevistados os responsáveis pela manutenção e condução da frota para observar os relatos dos mesmos quanto aos aspectos resultantes da substituição do óleo diesel pelo B5. Segundo uma avaliação do consumo de combustível, houve um aumento não-linear $(\approx 5 \%)$ quando da substituição do diesel pelo biodiesel B5, mas não foi observada qualquer manutenção adicional relacionada com a sua utilização; todavia foi compensado pela redução de emissões de compostos químicos de dióxido de nitrogênio e teor de enxofre, como medido por Fernandes (2011). Além disso, a funcionalidade e a vida residual em serviço dos ônibus utilizando misturas diesel são discutidos.

PALAVRAS-CHAVE: Frota de Ônibus Urbano e Interurbano, Motor Diesel, Biodiesel, Manutenção.

\section{EXPERIMENTAL EVALUATION OF THE SENSIVITY OF BIODIESEL B5 IN DIESEL POWERTRAIN OF THE URBAN AND INTERCITY BUS COMPANIES}

\begin{abstract}
The study aims to investigate the successful way of the biodiesel fuel used in the engines of urban and intercity bus companies. It was necessary to investigate and analyze, technologically, if the using biodiesel fuel presents problems relative to wear of parts directly involved with fuel and also to observe consumption fluctuations of this fuel. Fossil diesel fuel and B5 biodiesel blends were analytically analyzed, including relative to sulfur content. Two urban and intercity bus companies, had 41 and 13 vehicles, respectively, which were investigated in this work. It was analyzed 2008 until 2010 and were interviewed the management of the
\end{abstract}

maintenance team of bus companies relative to aspects concerning to substitution of the diesel fuel by the B5 biodiesel. According to consumption evaluation, there was a nonlinear boost $(\approx 5 \%)$ in the consumption of fuel when diesel was changed to B5 biodiesel blend, but it not was observed any additional maintenance related to use of this last fuel. This was rewarded by the emission reductions of chemical compound nitrogen dioxide and sulfur content, as measured by Fernandes (2011). Also, the functionality and residual life in service of the buses using diesel blends are discussed.

KEYWORD: Urban bus, intercity bus, Diesel powertrain, Biodiesel, Maintenance. 


\section{AVALIAÇÃO EXPERIMENTAL DA SENSIBILIDADE DO BIODIESEL B5 EM MOTORES DIESEL UTILIZADO EM FROTAS DE ÔNIBUS URBANO E INTERURBANO}

\section{INTRODUÇÃO}

Os estudos da Tribologia, ciência que estuda o desgaste, o atrito, a lubrificação e a vida de sistemas, associam-se a uma forte interação com o meio-ambiente. Quando os anéis de segmento de um pistão de um motor veicular se desgastam a uma taxa mais severa, devido à baixa lubricidade do combustível, ineficácia do sistema de lubrificação ou sua prolongada vida em serviço, por exemplo, um fluxo de fluidos de exaustão da combustão e material particulado fino $(<2,5 \mu \mathrm{m})$ é expelido a taxas crescentes para o meio ambiente.

No caso dos ônibus urbanos, esse problema se agrava, por se tratar de um meio de transporte definido para circular onde houver massificação de pessoas. E os tempos de exposição e as concentrações dessas emissões e outros materiais particulados vêm sendo investigados, como relata o estudo de Kaur e Nieuwenhuijsen (2007) no interior de ônibus em Londres, sobre a presença de monóxido de carbono e particulados finos em microambientes de transportes urbanos nos anos 90 .

Este trabalho se alinha nessa direção, no que diz respeito à percepção do seu uso em frotas de ônibus. Nas condições descritas, os óleos vegetais têm atraído as atenções como fonte renovável em potencial para produção de biodiesel como alternativa para substituir o diesel convencional (Costa Neto, 2000).

Em estudo promovido por Ahouissoussi e Wetzstein (1997) e associado a uma missão do Banco Mundial, uma simulação foi efetuada utilizando o método, desenvolvido por Rust (1987), para determinação de custos com manutenção de ônibus usando combustíveis alternativos, comparando os custos operacionais para sistemas de transporte por ônibus utilizando quatro tipos de combustíveis: biodiesel, gás natural comprimido, metanol e diesel convencional.

Os resultados dessa simulação de Ahouissoussi e Wetzstein (1997) indicaram que, apesar dos custos mais elevados, o Biodiesel puro e misturas Diesel-Biodiesel apresentavam o potencial de concorrer com o gás natural comprimido e o metanol como combustível para ônibus urbanos. Em sua simulação constavam duas planilhas, uma de custos tangíveis, integrada pelo dispêndio com (1) o combustível, (2) a manutenção e (3) a infraestrutura necessária à sua implantação e outra, de custos intangíveis, composta por diferentes níveis de confiabilidade dos motores de ônibus acionados pelos diferentes combustíveis investigados.

Esses autores admitiram que a determinação do custo de manutenção pressupõe a confiabilidade do funcionamento de diversos subconjuntos e peças individuais, cada um com probabilidade de falha aleatória em função da vida em serviço. Em sua simulação, foram consideradas as condições desde a substituição de peças até a troca do motor por um novo, após defeitos que implicariam na probabilidade de parada por falha na estrada em função de determinada quilometragem otimizada pelo custo, adequação funcional ao uso e confiabilidade operacional.

Derivado de fontes renováveis, o biodiesel é um combustível biodegradável, que pode ser obtido por diferentes processos (craqueamento, esterificação ou transesterificação). Assim, apenas o processo de transesterificação leva ao produto biodiesel, ou ésteres alquílicos de óleos e gorduras (Knothe et al., 2006), pois neste processo haverá redução da viscosidade dos triglicerídeos melhorando suas propriedades físico-químicas e otimizando-se assim seu uso como combustível (Silva, 2008). 
O biodiesel tem todas as características necessárias para substituir o diesel mineral nos motores a Diesel, com a vantagem adicional de ser virtualmente livre de enxofre e de compostos nocivos ao ser humano [Haas et al. (2001), Bagley et al. (1998)] e, segundo observações de Ahouissoussi e Wetzstein (1997), esta substituição proporciona modificações nos setores energético, econômico e ambiental - pois não contribui para o efeito estufa - o Biodiesel recicla carbono.

De acordo com Farias et. al. (2010), o biodiesel também possui melhor lubricidade que o diesel mineral. A lubricidade é de fundamental importância para o poder de lubrificação de uma substância, sendo uma função de várias de suas propriedades físicas, destacando-se principalmente a viscosidade e a tensão superficial. Possidonio (2008) destaca também que, diferentemente dos motores movidos à gasolina, os motores do ciclo Diesel exigem que o seu combustível tenha propriedades de lubrificação, especialmente em razão do funcionamento da bomba injetora, ou seja, em escoamento, possa lubrificar suas peças em movimento relativo.

Na sua forma pura ou como aditivo ao combustível diesel, o biodiesel pode ser usado para melhorar suas propriedades. Embora o biodiesel apresente uma quantidade de energia cerca de $10 \%$ menor que o diesel fóssil, seu desempenho no motor é praticamente o mesmo no que diz respeito à potência e ao torque (Agarwal et al., 2008). E apesar deste promover a redução da poluição do meio ambiente e melhorar a lubricidade das partes móveis no sistema de alimentação e injeção, ocorrerá um aumento do processo de desgaste destes componentes [Bovington et al. (1994), Martins (2005)].

Em um artigo de revisão da literatura, Knothe et al. (1997) asseguram que as misturas de Biodiesel, ao final do Século $X X$, avançavam da condição de combustíveis puramente experimentais para os estágios iniciais de comercialização por serem tecnicamente competitivos, embora permanecessem problemas de combustão, econômicos, de contaminação do lubrificante e de suas propriedades às baixas temperaturas. Um exemplo desses problemas que afetam o sistema de alimentação de combustível é o bico injetor uma peça importante sujeita ao desgaste. Segundo estudo de Oliveira Filho (2011), durante o funcionamento do bico injetor, acontece a formação do desgaste adesivo por clogging.

O setor de transportes tem como característica própria a elevada demanda de combustíveis fósseis (derivados de petróleo). Um dos grandes desafios da sociedade contemporânea é o desenvolvimento de novos combustíveis e fontes de energia que possam substituir esses derivados, independentemente de sua oferta atual e futura. As principais forças mobilizadoras para esse desenvolvimento passam pela racionalização do uso dos recursos naturais disponíveis, buscando favorecer a sustentabilidade em todas as dimensões, seja energética, econômica, social ou ambiental (Petrobras, 2011).

A Empresa de Pesquisa Energética (EPE) do MME (Ministério das Minas e Energia) prevê aumento do consumo de energia no Brasil; em especial, o aumento da demanda do setor de transportes. Pode-se observar, na Fig. 1, que esse setor, atualmente responsável por $30 \%$ do consumo energético do país, deverá ter sua participação incrementada em 4\%, até 2030 (Rezende, 2009).

2005

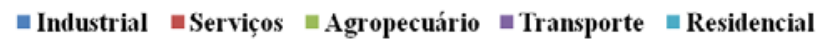

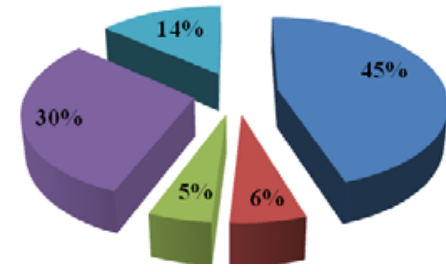

2030

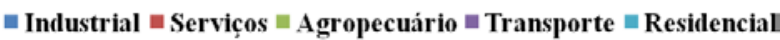

Obs.: exclusive consumo não energético
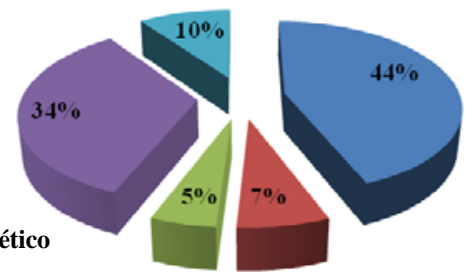
Frota de veículo no Brasil em 2007 - Total: 25,6 milhões (2,5\% do mundo)

Figura 1 - Estrutura, por setor, do consumo de energia no Brasil, adaptado de Rezende (2009)

Este trabalho trata de um estudo experimental sobre a influência do uso do biodiesel B5 em relação ao diesel comum utilizado por duas frotas de ônibus, urbana e outra interurbana, com veículos variando de 1 a 23 anos de uso, as quais pertencem a empresas sediadas em Natal - RN, Brasil. A percepção e/ou o registro da manutenção dos respectivos motores foram efetuados quando estes veículos passaram a utilizar Biodiesel B5.

\section{MATERIAIS E MÉTODOS}

\section{Caracterização dos Combustíveis Utilizados}

O combustível B5 (blenda com $5 \%$ em volume de biodiesel e $95 \%$ de diesel puro), utilizado no abastecimento da frota de ônibus urbano da empresa Transporte Cidade do Natal Ltda, é fornecido pela BR Distribuidora e ALESAT Combustíveis S.A.; enquanto que a frota de ônibus interurbano, da Auto Viação Jardinense, é abastecida pelo combustível B5 da Transcopel Distribuidora. Amostras destes combustíveis foram cedidas pelas referidas empresas de transporte, objetivando-se a sua caracterização físico-química. $O$ diesel puro foi como utilizado como referencial padrão, cuja obtenção deu-se junto à Petrobras S.A. via NUPEG II (Núcleo de Pesquisa em Petróleo e Gás II) da UFRN.

As características físico-químicas avaliadas para o biodiesel B5 e o diesel puro foram: Aspecto visual, Viscosidade cinemática à $40{ }^{\circ} \mathrm{C}$ (ASTM D 445), Massa específica à $15^{\circ} \mathrm{C} / 20$ 으 (ASTM D 4052), Teor de Enxofre (ASTM D 4294), Índice de Acidez (ASTM D 664), Ponto de Fulgor (ASTM D 93). Esta caracterização foi executada no Laboratório de Combustíveis e Lubrificantes da UFRN e os valores obtidos estão indicados na Tab. 1.

Tabela 1: Caracterização físico-química dos combustíveis

\begin{tabular}{|l|c|c|c|c|}
\hline \multirow{2}{*}{\multicolumn{1}{|c|}{ Propriedades }} & \multirow{2}{*}{ Limite } & \multicolumn{2}{c|}{ Biodiesel B5 } & Diesel puro \\
\cline { 3 - 5 } & LII & $\begin{array}{c}\text { Transporte Cidade do } \\
\text { Natal Ltda }\end{array}$ & $\begin{array}{c}\text { Auto Viação } \\
\text { Jardinense }\end{array}$ & $\begin{array}{c}\text { Petrobras via } \\
\text { NUPEG II }\end{array}$ \\
\hline Aspecto & TII & TII & TCI \\
\hline Cor & - & Vermelho & Vermelho & Amarelo \\
\hline $\begin{array}{l}\text { Massa Específica, } 15^{\circ} \mathrm{C}, \\
\mathrm{kg} / \mathrm{m}^{3}\end{array}$ & 841 & 842 & 824 \\
\hline $\begin{array}{l}\text { Massa Específica, } 20^{\circ} \mathrm{C}, \\
\mathrm{kg} / \mathrm{m}^{3}\end{array}$ & 820 a 880 & 837 & 839 & 821 \\
\hline \begin{tabular}{l} 
Ponto de Fulgor, ${ }^{\circ} \mathrm{C}$ \\
\hline Enxofre, $\mathrm{mg} / \mathrm{kg}$
\end{tabular} & 38 & 42 & 43 & 37 \\
\hline $\begin{array}{l}\text { Índice de Acidez, } \mathrm{mg} \\
\mathrm{KOH} / \mathrm{g}\end{array}$ & - & 970 & 1230 & 1110 \\
\hline $\begin{array}{l}\text { Viscosidade cinemática } \\
\text { a } 40^{\circ} \mathrm{C}, \mathrm{cSt}-\mathrm{mm}^{2} / \mathrm{s}\end{array}$ & 2,0 a 5,0 & 0,66 & 0,62 & 0,38 \\
\hline
\end{tabular}

Metodologias Aplicadas na Avaliação do Desempenho de Misturas de Biodiesel em Motores Diesel 
Objetivando-se analisar a sensibilidade do consumo do combustível em relação à influência na mudança do diesel comum (diesel puro) pelo B5, selecionaram-se duas empresas, uma de transporte urbano, a Transporte Cidade do Natal Ltda, e outra, de transporte interurbano, a Auto Viação Jardinense. Destas empresas foram obtidos dados referentes ao ICC (Índice de Consumo de Combustível) dos 54 ônibus, em um período compreendido entre setembro de 2008 e julho de 2010.

A frota da empresa Transporte Cidade do Natal Ltda compõe-se 41 ônibus dos fabricantes Mercedes-Benz (modelo OF 1418, OF 1722) e Volkswagen (Volksbus VW 15180, VW 15190, VW 17210). Já a frota de ônibus interurbanos da empresa Auto Viação Jardinense é composta por 13 veículos dos fabricantes Mercedes-Benz (OM 355/5A, OM 355/6, OM 447/A) e Volvo (B10M THD $101 \mathrm{KC}$ ). A configuração das frotas utilizadas para a obtenção dos resultados de ICC é especificada na Tab. 2.

Tabela 2: Configuração das frotas de transporte urbano e interurbano de passageiros

\begin{tabular}{|c|l|}
\hline Frota de estudo & \multicolumn{1}{|c|}{ Transporte Cidade do Natal Ltda. } \\
\hline & Ônibus Mercedes-Benz OF 1418 com motor OM904 LA 130 kW e \\
OF 1722 com motor OM924 LA 160 kW ambos equipados com \\
(Transporte coletivo) & $\begin{array}{l}\text { UPS (unit pump system); Volksbus VW 15180 com motor MWM } \\
\text { 6.12 ODE, VW 15190 com motor MWM 4.12 ODE e VW 17210 } \\
\text { com motor MWM 6.10 TCA }\end{array}$ \\
\hline Tipo de carga da frota urbana & Passageiros \\
\hline Frota de estudo & \multicolumn{1}{|c|}{ Auto Viação Jardinense } \\
\hline Veículos & $\begin{array}{l}\text { Ônibus Mercedes-Benz Modelos OM 355/5A, OM 355/6, OM } \\
\text { 447/A; Volvo B10M THD 101 KC }\end{array}$ \\
\hline Transporte coletivo) & Passageiros e bagagens \\
\hline Tipo de carga da frota interurbana
\end{tabular}

Análise da Frota de Ônibus Urbano Transporte Cidade do Natal Ltda

A tabela 3 refere-se a caracterização dos motores com admissão utilizando turbo/intercooler, os quais equipam todos os veículos da frota pertencente à empresa Transporte Cidade do Natal Ltda. Esta frota é composta por 41 veículos, sendo 12 da marca Volkswagen e 29 da marca Mercedes-Benz.

Tabela 3: Caracterização dos tipos de motores utilizados na frota urbana

\begin{tabular}{|c|c|c|c|c|c|c|c|c|}
\hline $\begin{array}{l}\text { № de } \\
\text { Ônibus }\end{array}$ & $\begin{array}{l}\text { Fabricante/ } \\
\text { Modelo }\end{array}$ & $\begin{array}{l}\text { Cilindrada/ } \\
\text { Potencia/ } \\
\text { Rotação } \\
\end{array}$ & $\begin{array}{c}\text { Válvulas/ } \\
\text { cilindro }\end{array}$ & $\begin{array}{l}\text { Sistema de } \\
\text { injeção }\end{array}$ & $\begin{array}{l}\text { Taxa de } \\
\text { comp. }\end{array}$ & $\begin{array}{c}\text { Pressão } \\
\text { de } \\
\text { injeção }\end{array}$ & $\begin{array}{c}\text { Tipo de } \\
\text { óleo }\end{array}$ & $\begin{array}{l}\text { Temp. de } \\
\text { trabalho }\end{array}$ \\
\hline 20 & $\begin{array}{l}\text { Mercedes- } \\
\text { Benz } \\
\text { OF } 1418\end{array}$ & $\begin{array}{c}4.249 \mathrm{~cm}^{3} \\
177 \mathrm{cV} \\
2200 \mathrm{RPM}\end{array}$ & $\begin{array}{c}3 \mathrm{~V} / \\
\text { Cilindro }\end{array}$ & $\begin{array}{c}\text { PLD - direta, } \\
\text { gerenciamento } \\
\text { eletrônico }\end{array}$ & $19,1: 1$ & $\begin{array}{c}200- \\
245 \text { Bar }\end{array}$ & \begin{tabular}{|c|} 
Lubrax \\
Top Turbo \\
15W40 \\
\end{tabular} & 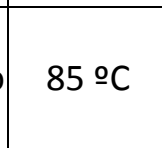 \\
\hline 9 & $\begin{array}{l}\text { Mercedes- } \\
\text { Benz } \\
\text { OF } 1722\end{array}$ & $\begin{array}{c}4.800 \mathrm{~cm}^{3} \\
218 \mathrm{cV} \\
2200 \mathrm{RPM}\end{array}$ & $\begin{array}{c}\text { 2V/ } \\
\text { Cilindro }\end{array}$ & $\begin{array}{l}\text { PLD - direta, } \\
\text { gerenciamento } \\
\text { eletrônico }\end{array}$ & $19,1: 1$ & $\begin{array}{c}200- \\
245 \text { Bar }\end{array}$ & \begin{tabular}{|c|} 
Lubrax \\
Top Turbo \\
15W40
\end{tabular} & 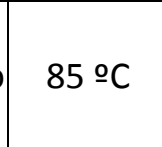 \\
\hline 6 & $\begin{array}{l}\text { Volkswagen } \\
\text { VW } 15190\end{array}$ & $\begin{array}{c}6.450 \mathrm{~cm}^{3} \\
189 \mathrm{cV} \\
2750 \mathrm{RPM} \\
\end{array}$ & $\begin{array}{c}\text { 2V/ } \\
\text { Cilindro }\end{array}$ & $\begin{array}{c}\text { Direta - } \\
\text { Common rail }\end{array}$ & 16,9: 1 & $\begin{array}{c}1350- \\
1600 \\
\text { Bar }\end{array}$ & \begin{tabular}{|c|} 
Lubrax \\
Top Turbo \\
$15 \mathrm{~W} 40$ \\
\end{tabular} & $90 \stackrel{\circ}{ } \mathrm{C}$ \\
\hline 1 & $\begin{array}{l}\text { Volkswagen } \\
\text { VW } 15180\end{array}$ & \begin{tabular}{|c|}
$7.120 \mathrm{~cm}^{3}$ \\
$177 \mathrm{cV}$ \\
$2750 \mathrm{RPM}$ \\
\end{tabular} & $\begin{array}{c}\text { 2V/ } \\
\text { Cilindro }\end{array}$ & $\begin{array}{c}\text { Direta - } \\
\text { Common Rail }\end{array}$ & 16,8: 1 & $\begin{array}{c}1350- \\
1600 \\
\text { Bar }\end{array}$ & \begin{tabular}{|c|} 
Lubrax \\
Top Turbo \\
$15 \mathrm{~W} 40$ \\
\end{tabular} & $90 \stackrel{\circ}{ }$ \\
\hline
\end{tabular}


OLIVEIRA FILHO ET AL. (2012)

\begin{tabular}{|c|c|c|c|c|c|c|c|c|}
\hline 5 & Volkswagen & $\begin{array}{c}6.450 \mathrm{~cm}^{3} \\
214 \mathrm{cv} 2600 \\
\text { RPM }\end{array}$ & $\begin{array}{c}2 \mathrm{~V} / \\
\text { Cilindro }\end{array}$ & $\begin{array}{c}\text { Direta - } \\
\text { Common Rail }\end{array}$ & $\begin{array}{c}1350-0: 1 \\
16210\end{array}$ & $\begin{array}{c}\text { Lubrax } \\
\text { Top Turbo } \\
15 \mathrm{~W} 40\end{array}$ & 90 oC \\
\hline
\end{tabular}

Na figura 2 são mostradas imagens dos veículos de marca Mercedes Benz, modelo OF 1418/2008, e o de marca VW, modelo 15190/2008 pertencente à empresa Transporte Cidade do Natal Ltda e sua infraestrutura de abastecimento de combustível, Fig. 3.

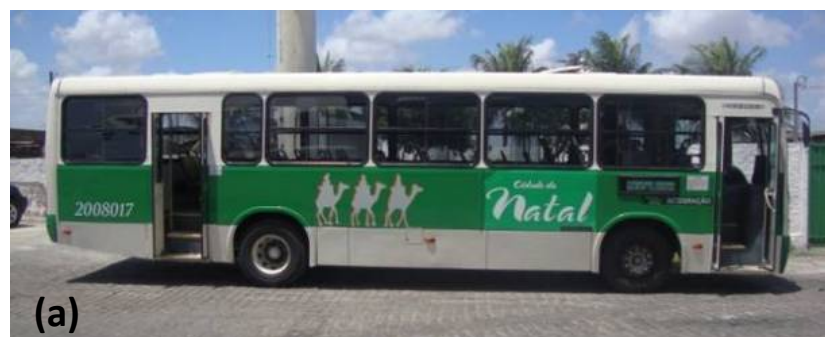

Figura 2 - Veículos modelos (a) OF 1418/ 2008 e (b) VW 15190/ 2008
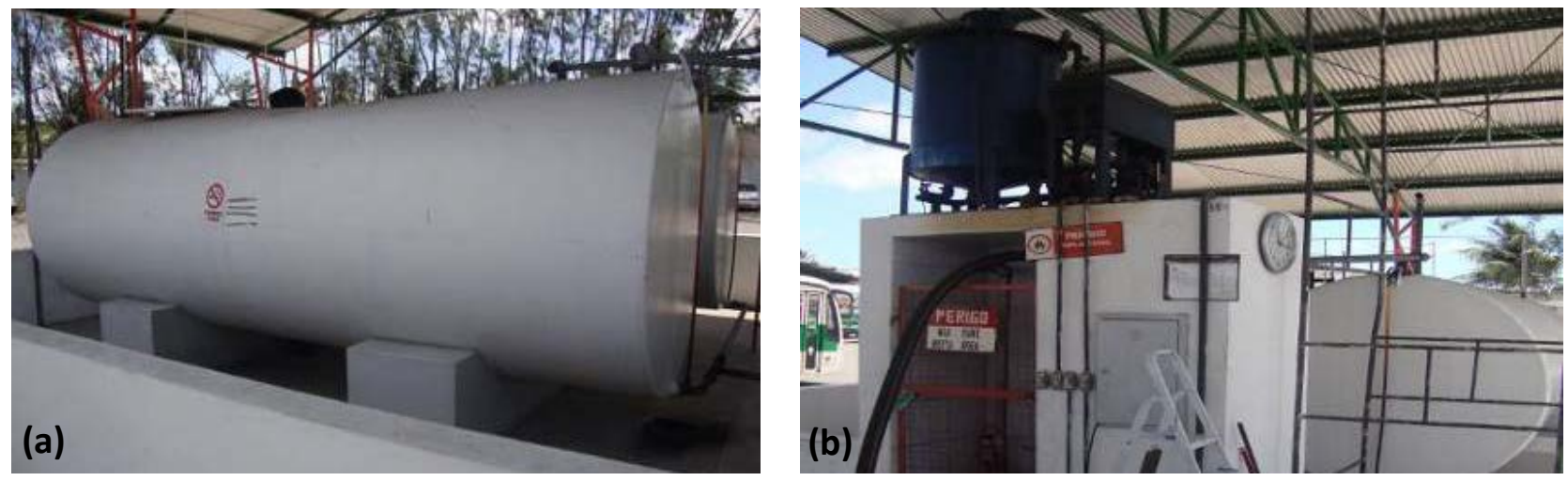

Figura 3 - Infraestrutura: (a) tanque reservatório e (b) bomba de abastecimento

Segundo a política de manutenção adotada por esta empresa, a sistemática da manutenção leva em conta algumas informações com relação ao perfil de operação dos seus veículos, dentre elas temos: (1) Média de giro mensal é cerca de 9.000 km; (2) Substituição do óleo do motor: é feita a cada 20.000 km; (3) Manutenção: do tipo corretiva, contudo o sistema preventivo está em fase de implantação.

\section{Análise da Frota de Ônibus Interurbano da Auto Viação Jardinense}

Todos os veículos da frota da empresa Auto Viação Jardinense utilizam motores com sistema de admissão turbo/intercooler, os quais são caracterizados na Tab. 4. Na figura 4 e 5 são exibidos o veículo Volvo B $10 \mathrm{M}$ THD 101 KC, sua motorização e a infraestrutura de abastecimento de combustível, respectivamente.

Tabela 4: Caracterização dos tipos de motores utilizados na frota interurbana

\begin{tabular}{|c|c|c|c|c|c|c|c|}
\hline $\begin{array}{c}\text { No de } \\
\text { Onnibus }\end{array}$ & $\begin{array}{c}\text { Fabricante/ } \\
\text { Modelo }\end{array}$ & $\begin{array}{c}\text { Cilindrada/ } \\
\text { Potencia/ } \\
\text { Rotação }\end{array}$ & $\begin{array}{c}\text { Sistema } \\
\text { de } \\
\text { injeção }\end{array}$ & $\begin{array}{c}\text { Taxa de } \\
\text { comp. }\end{array}$ & $\begin{array}{c}\text { Pressão de } \\
\text { injeção }\end{array}$ & $\begin{array}{c}\text { Tipo de } \\
\text { óleo }\end{array}$ & $\begin{array}{c}\text { Temp. de } \\
\text { trab. }\end{array}$ \\
\hline 6 & $\begin{array}{c}\text { Mercedes- } \\
\text { Benz } \\
\text { OM 355/5 A }\end{array}$ & $\begin{array}{c}9.651 \mathrm{~cm}^{3} \\
238 \mathrm{cV} \\
2100 \mathrm{RPM}\end{array}$ & $\begin{array}{c}\text { Injeção } \\
\text { Direta }\end{array}$ & $\begin{array}{c}16: 1 \\
200 \text { a } 245 \\
\text { Bar }\end{array}$ & $\begin{array}{c}\text { Lubrax } \\
\text { Top Turbo } \\
15 W 40\end{array}$ & $87^{\circ} \mathrm{C}$ \\
\hline
\end{tabular}


OLIVEIRA FILHO ET AL. (2012)

\begin{tabular}{|c|c|c|c|c|c|c|c|}
\hline 1 & $\begin{array}{c}\text { Mercedes- } \\
\text { Benz } \\
\text { OM 447/ A }\end{array}$ & $\begin{array}{c}11.967 \mathrm{~cm}^{3} \\
333 \mathrm{cv} \\
1800 \mathrm{RPM} \\
\end{array}$ & $\begin{array}{l}\text { Injeção } \\
\text { Direta }\end{array}$ & $16,25: 1$ & $\begin{array}{c}200 \text { a } 245 \\
\text { Bar }\end{array}$ & \begin{tabular}{|c|}
$\begin{array}{c}\text { Lubrax } \\
\text { Top Turbo } \\
\text { 15W40 }\end{array}$ \\
\end{tabular} & $85^{\circ} \mathrm{C}$ \\
\hline 2 & $\begin{array}{c}\text { Mercedes- } \\
\text { Benz } \\
\text { OM } 355 / 6 \mathrm{~A} \\
\end{array}$ & $\begin{array}{c}11580 \mathrm{~cm}^{3} \\
189 \mathrm{cV} \\
2000 \mathrm{RPM} \\
\end{array}$ & $\begin{array}{l}\text { Injeção } \\
\text { Direta }\end{array}$ & $16: 1$ & $\begin{array}{c}1350 \text { a1600 } \\
\text { Bar }\end{array}$ & \begin{tabular}{|c|} 
Lubrax \\
Top Turbo \\
$15 \mathrm{~W} 40$ \\
\end{tabular} & $90^{\circ} \mathrm{C}$ \\
\hline 4 & $\begin{array}{c}\text { Volvo } \\
\text { B10M THD } \\
101 \mathrm{KC}\end{array}$ & $\begin{array}{c}9.600 \mathrm{~cm}^{3} \\
310 \mathrm{cv} \\
2200 \mathrm{RPM}\end{array}$ & $\begin{array}{l}\text { Injeção } \\
\text { Direta }\end{array}$ & $15: 1$ & $\begin{array}{c}1350-1600 \\
\text { Bar }\end{array}$ & \begin{tabular}{|c|} 
Lubrax \\
Top Turbo \\
$15 W 40$
\end{tabular} & $85^{\circ} \mathrm{C}$ \\
\hline
\end{tabular}
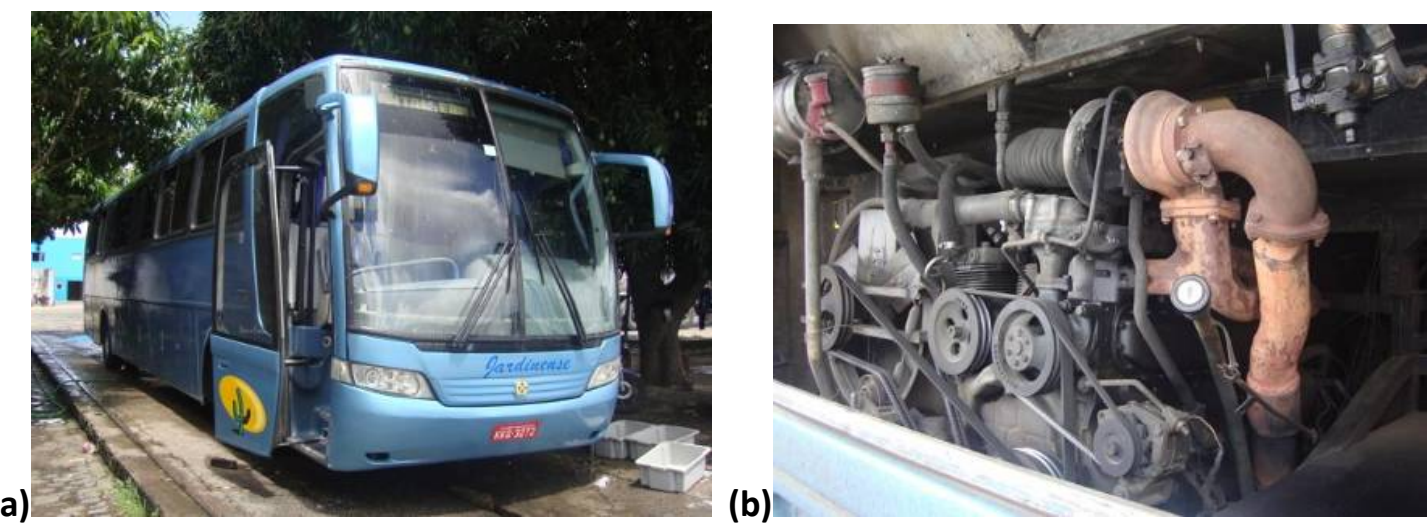

Figura 4 - (a)Veículo Volvo B 10M THD 101 KC e (b) motorização

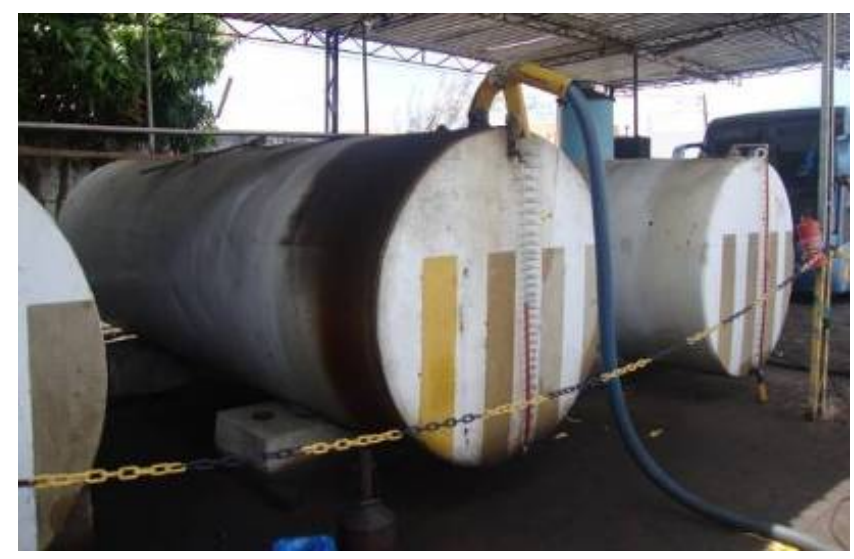

Figura 5 - Infraestrutura de abastecimento de combustível

Semelhante à sistemática de manutenção da Transporte Cidade do Natal Ltda, a Auto Viação Jardinense possui um perfil de operação dos seus veículos com as seguintes considerações: (1) Média de giro mensal é cerca de 9.000 km; (2) Óleo do motor e filtro trocados a cada 20.000 km e; (3) Manutenção: corretiva.

\section{RESULTADOS E DISCUSSÃO}

As informações a seguir são referentes aos resultados de consumo médio de combustíveis (índice de consumo de combustível - ICC), considerando os fabricantes e modelos de veículos pertencentes às frotas de ônibus urbana e interurbana, bem como observações adicionais por parte dos responsáveis pela manutenção destas frotas considerando os aspectos resultados da política brasileira ambientalmente amigável para a substituição do óleo diesel pelo B5 em suas frotas no ano de 2010. 


\section{Sistematização da Avaliação do ICC da Frota Urbana}

Foi adquirido registro de ICC e quilometragem dos veículos da frota urbana, da empresa Transporte Cidade do Natal Ltda, referentes ao período contínuo de setembro de 2008 a julho de 2010, em que pôde-se efetuar a análise do perfil de mudança dos índices de combustível desse período, como são observadas na Fig. 6, considerando o fabricante/modelo de ônibus utilizado.

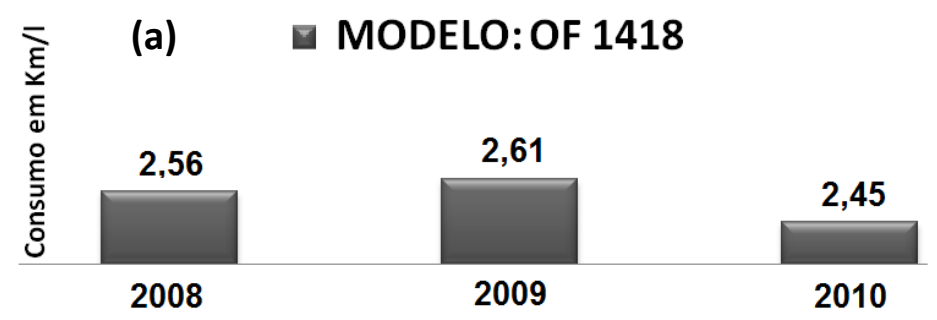

(b) $\square$ MODELO: VW 15180

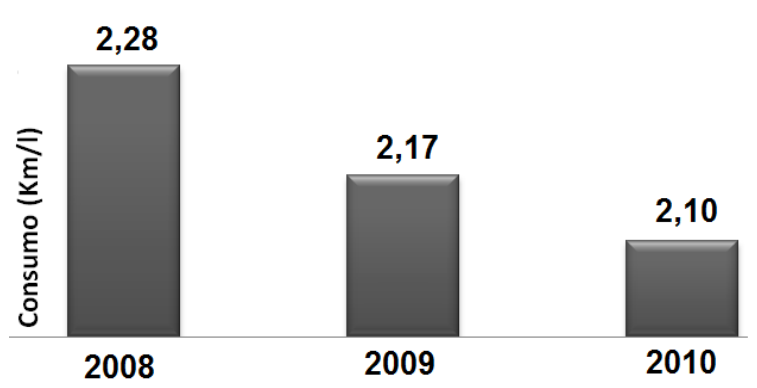

(d) $\quad \square$ MODELO: VW 15190

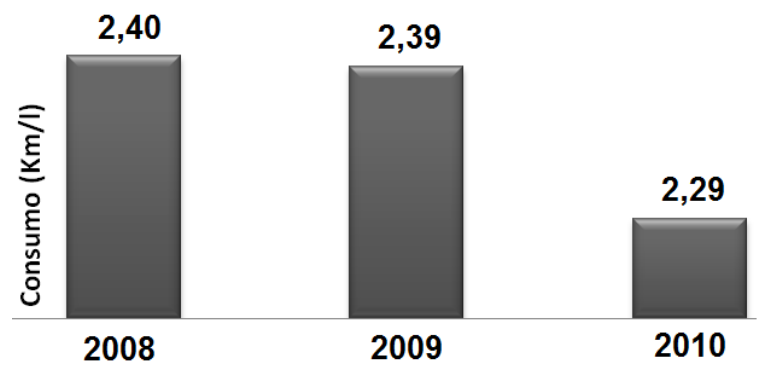

(c) $\square$ MODELO:VW 17210

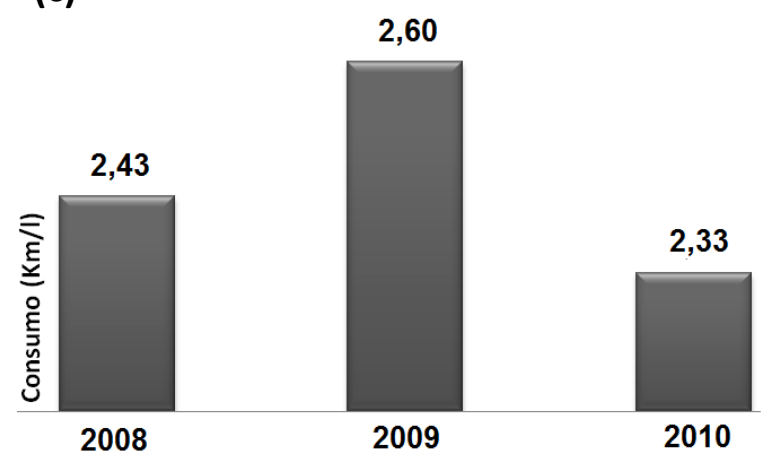

(e) $\square$ MODELO: OF 1722

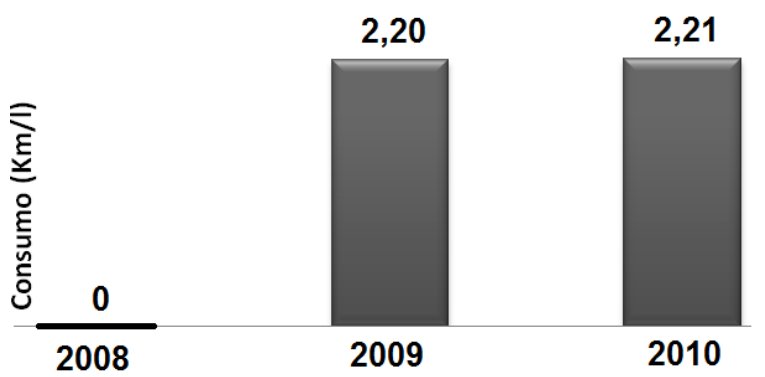

Figura 6 - Consumo médio de combustível (ICC) para os modelos (a) OF 1418, (b) VW 17210, (c) VW 15190, (d) VW 15180 e, (e) OF 1722

Na Figura 7, é indicada a avaliação dos dados de utilização da frota de ônibus urbanos, efetuando-se um comparativo entre os modelos, no período 2008 a 2010. 


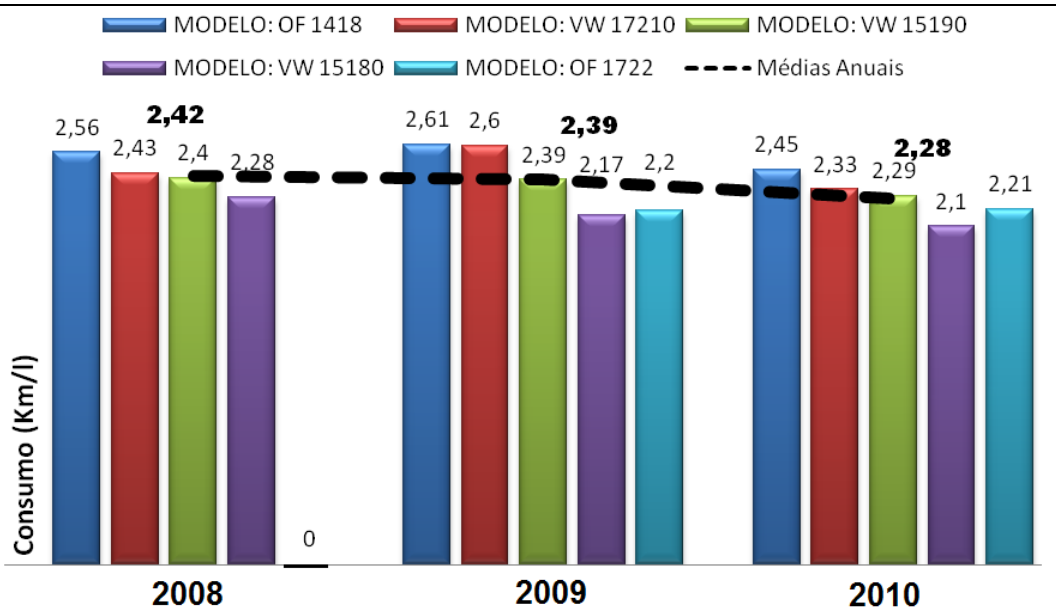

Figura 7 - ICC médio comparativo dos modelos, onde o consumo "zero" em 2008 diz respeito aos ônibus novos incorporados à frota a partir de 2009

Conforme observado na Fig. 7, considerando a transição do combustível diesel mineral para o B5, ao longo desses anos houve um aumento não linear de consumo de combustível de aproximadamente $5 \%$, em função da queda na potência dos motores em decorrência do biodiesel ter um menor poder calorífico. Nota-se também que o veículo modelo VW 15180 possui o maior consumo em relação aos demais modelos, e que aumenta seu ICC de $2,28 \mathrm{~km} / \mathrm{L}$ (em 2008) para 2,1 km/L (em 2010), enquanto os 20 veículos modelo OF 1418 apresentaram o menor consumo para os mesmos períodos, apresentando maior ICC médio em 2009 de 2,61 km/L e, após validação do B5, 2,45 km/L.

Segundo o supervisor de manutenção desta empresa, após o uso do biodiesel, observaram-se os seguintes fatos:

I. houve um moderado aumento de falhas em alguns veículos com o sistema de injeção eletrônica da VW. Entretanto, este diz não poder afirmar se a causa do aumento dos problemas seria pelo uso do biodiesel.

II. não se constatou diferença significativa na manutenção dos motores usando o biodiesel em relação ao período anterior, caracterizado pelo uso do diesel puro.

\section{Sistematização da avaliação do ICC na frota interurbana}

Foi adquirido registro de ICC e quilometragem dos veículos interurbanos, pertencentes à Auto Viação Jardinense, referentes a duas séries descontínuas de tempo. O primeiro período incluía outubro de 2008 a abril de 2009 e, o segundo, de janeiro a agosto de 2010. Esses dados estão esboçados na Fig. 8.

(a) $\square$ MODELO:OM 355/5 A

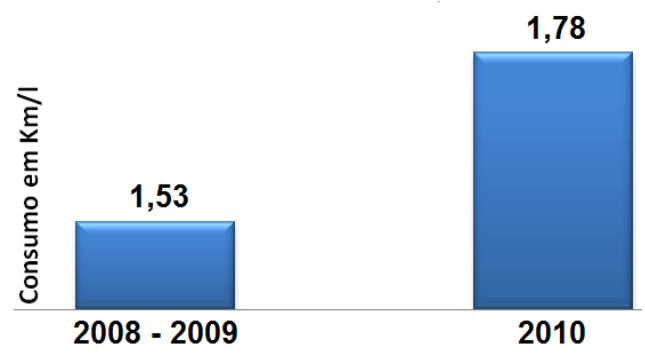

(c) (b) $\square$ MODELO:B 10M THD $101 \mathrm{KC}$

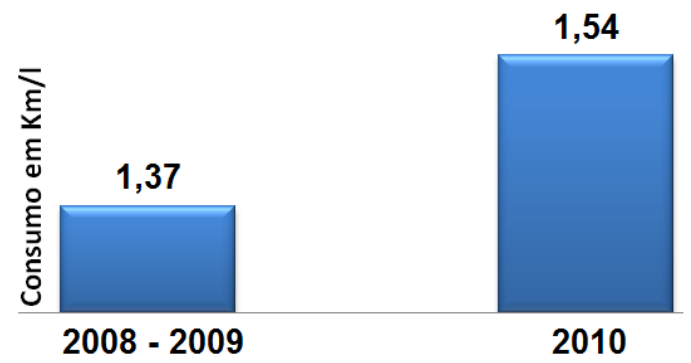

(d) 


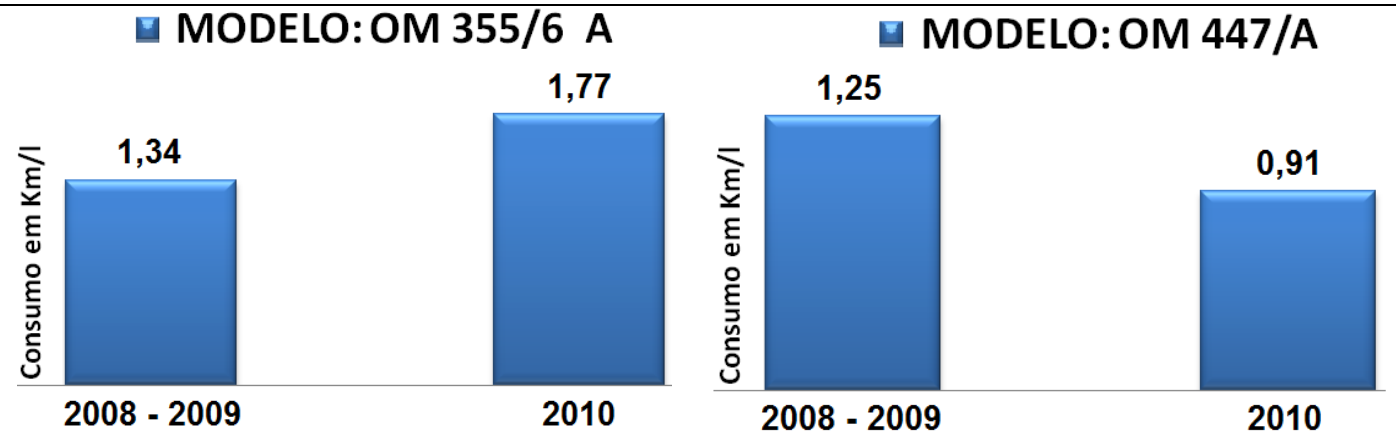

Figura 8 - Consumo médio (ICC) de combustível para os modelos (a) OM 355/5 A, (b) B 10M THD 101 KC, (c) OM 355/6 e, (d) OM 447/A

Na figura 9, é indicado um comparativo do consumo, no período de 2008 a 2010, entre os modelos veiculares da Auto Viação Jardinense. Uma análise mais detalhada do gráfico desta figura tornou-se prejudicada, tendo em vista a descontinuidade das informações disponibilizadas. Contudo, nota-se, nos gráficos comparativos, que o ICC médio aumentou de 1,38 (de 2008 a 2009) para 1,42 km/L, em 2010. Assim, o veículo modelo OM 447/A apresentou maior aumento de consumo de combustível (ICC $=0,91 \mathrm{~km} / \mathrm{L}$ ) que os demais dessa frota, enquanto os seis veículos modelo OM 355/5 A apresentaram menor consumo (ICC = 1,70 km/L), após a troca pelo B5.

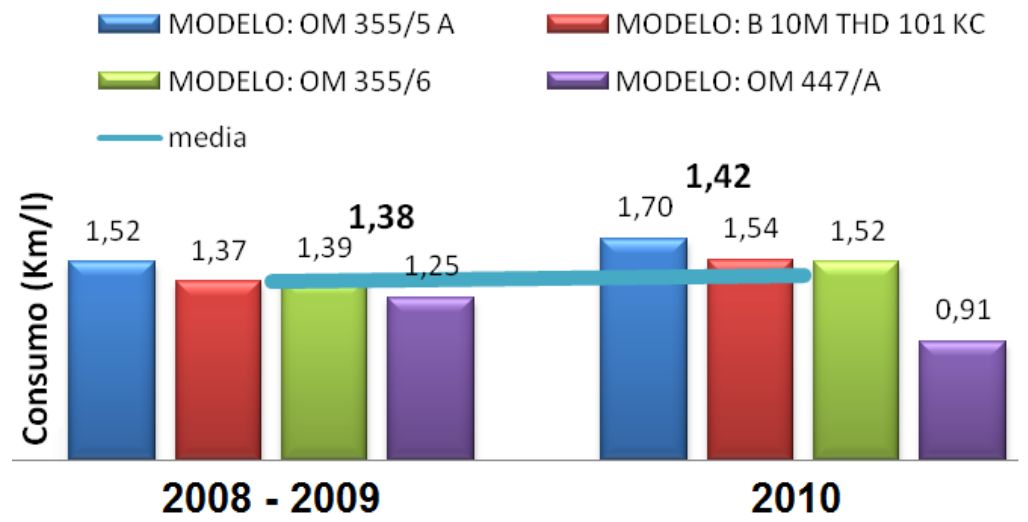

Figura 9 - ICC médio comparativo dos modelos

Ainda, de acordo com observações do pessoal da manutenção dessa empresa, eles afirmam que (I) "não há ninguém que opere os veículos e que tenha percepção de associar o desempenho do biodiesel em relação ao diesel" e (II) "a manutenção utilizada, no período, tem sido completamente corretiva".

De uma forma geral, observa-se, para o consumo de combustível da frota urbana, que houve um aumento não-linear (cerca de $5 \%$ ) quando da substituição do diesel pelo biodiesel B5, entretanto não foi observada qualquer manutenção adicional em decorrência de sua utilização, durante o período de realização desta pesquisa. Entretanto, para ambas as frotas, a troca do combustível de origem fóssil pelo B5 foi positiva sob o aspecto da redução de emissões de compostos químicos de dióxido de nitrogênio e teor de enxofre, como medido por Fernandes (2011) em sua tese realizada no NUPEG II do PPGEQ-UFRN (Programa de PósGraduação em Engenharia Química da UFRN). 


\section{CONCLUSÃO}

O trabalho desenvolvido permitiu que se comparasse o desempenho de motores ciclo Diesel utilizando diesel e a mistura do Biodiesel (B5), em duas frotas de ônibus urbana e interurbana, ambas com sede na cidade de Natal-RN, Brasil. Pode-se concluir que:

- Foi constatado que, com a mudança do combustível de diesel mineral para biodiesel (B5 ou B20), houve uma diminuição nas emissões dos dióxidos de nitrogênio e de enxofre, bem como nas emissões de hidrocarbonetos, de extrema importância ambiental;

- Registrou-se, em linhas gerais, que houve um aumento do consumo de combustível e uma diminuição em termos de potência quando se migrou do diesel puro para a mistura B5;

- No período de desenvolvimento do trabalho não se registraram anomalias na manutenção dos motores dos ônibus associados quando da migração para o uso do B5, conforme relatório da gerência de manutenção;

- Os veículos de ambas as frotas urbana e interurbana com melhor ICC - índice de consumo de combustível médio (em km/L) em 2010 foram os de modelo OF 1418 (2,45 km/L) e OM 355/5 A $(1,70 \mathrm{~km} / \mathrm{L})$, respectivamente, enquanto que os veículos com maiores consumos de combustível das frotas urbana e interurbana foram, nesta ordem, o de modelo VW $15180 \mathrm{com}$ ICC médio de 2,1 km/L e o de modelo OM 477/A, com ICC médio de 0,91 km/L, ambos em 2010.

\section{REFERÊNCIAS BIBLIOGRÁFICAS}

1. AGARWAL, D.; KUMAR, L.; AGARWAL, A.K. Performance evaluation of a vegetable oil fuelled compression ignition engine. Renewable Energy, 33, p.1147-1156, 2008.

2. AHOUISSOUSSI, N.B.C.; WETZSTEIN, M.E. A comparative cost analysis of Biodiesel, compressed natural gas, methanol, and diesel for transit bus systems. Resource and Energy Economics 20, p.1-15, 1997.

3. BAGLEY, S.T.; GRATZ, L.D.; JOHNSON, J.H.; MCDONALD, J.F. Termoquímica da reação de autooxidação do biodiesel de óleo de soja: um estudo teórico. Environ. Sci. Technol., 32, n. 1183, 1998.

4. BOVINGTON, C., CAPROTTI, R., MEYER, K., and SPIKES, H. A. Development of a laboratory test to predict properties of diesel fuels and its application to the development of highly refined diesel fuels. In: International Colloquium, Ecological and Economic Aspects of Tribology, 9, 1994, Esslingen, Germany. Anais Esslingen, Germany, 1994.

5. COSTA NETO, P.R. Produção de biocombustíveis alternativo ao óleo diesel através da transesterificação de óleo de soja usado em frituras. Revista Química Nova, 23 (4), p. 531$537,2000$.

6. FARIAS, A. C. M.; SANTANA, J.S.; OLIVEIRA FILHO, M. F.; SANTANA, J.S.; BARBOSA, C.R.F.; MEDEIROS, J.T.N. Os combustíveis verdes do Brasil - Avaliação da lubricidade do óleo biodiesel B5 e óleos vegetais de coco e mamona. Anais do VI Congresso Nacional de Engenharia Mecânica, CONEM 2010. Campina Grande - PB, 2010.

7. FERNANDES, M.R. Avaliação do desempenho e das emissões de um motor ciclo diesel utilizando sistemas diesel/água/biodiesel/óleo vegetal. Tese apresentada à Universidade Federal do Rio Grande do Norte, Natal-RN, Brasil, 2011, 136p. 
8. HAAS, M. J.; SCOTT, K. M.; ALLEMAN, T.L.; MCCORMICK, R.L. Engine performance of biodiesel fuel prepared from soybean soapstok a high quality renewable fuel produced from a waste feedstock. Energy Fuels, 15 (5), p. 1207, 2001.

9. KAUR, S.; NIEUWENHUIJSEN, M.J.; COLVILE, R.N. Fine particulate matter and carbon monoxide exposure concentrations in urban street transport microenvironments. Atmospheric Environment 41, p. 4781-4810, 2007.

10. KNOTHE, G.; GERPEN, J.V.; KRAHL, J.; RAMOS, L.P. Manual de biodiesel. São Paulo, Editora Edgard Blücher, 2006.

11. KNOTHE, G.; DUNN, R.O.; BAGBY, M.O. Biodiesel: The use of vegetable oils and their derivatives as alternative diesel fuels. In: ACS Symposium Series No. 666: Fuels and chemicals from biomass. Washington DC, USA, p. 172-208, 1997. Disponível em: http://www.biodieselgear.com/documentation/VegetableOilsAsAlternativeDieselFuels.pdf. Acesso em 02 janeiro 2011.

12. MARTINS, J. Motores de combustão interna. 1.ed., Portugal: Publindústria Edições Técnicas, 2005, s.p.

13. PETROBRAS. Cronograma do acordo sobre a distribuição do diesel S-50. Disponível em http://ww.agenciapetrobrasdenoticias.com.br. Acesso em 20 de janeiro de 2011.

14. POSSIDONIO, E. R. Otimização das condições de síntese de biodiesel de óleo de soja, visando atender normas de qualidade da ANP. Fundação de Estudos e Pesquisas Agrícolas e Florestais, 2008, 33 p.

15. OLIVEIRA FILHO, M.F. Análise da sensibilidade do biodiesel B5 em frotas de transporte coletivo com motorização diesel. Dissertação de mestrado apresentada à Universidade Federal do Rio Grande do Norte, Natal-RN, Brasil, 2011, 111p.

16. REZENDE, S. Energias alternativas para propulsão veicular: pesquisa e desenvolvimento no Brasil. Estudos e pesquisas no 304. Anais do XXI Fórum Nacional - Na Crise Global, o Novo Papel Mundial dos BRICs (BRIMCs?) e as Oportunidades do Brasil (Crise como Oportunidade, Através do Plano de Ação), Rio de Janeiro/ RJ, 2009.

17. RUST, J. Optimal replacement of GMC bus engines: An empirical model of Harold Zurcher, Econometrica 55, p. 999-1033, 1987.

18. SILVA, L.L. Estudos de óleos residuais oriundos de processo de fritura e qualificação desses para obtenção de monoésteres (biodiesel). Dissertação de mestrado apresentada à Universidade Federal de Alagoas, Brasil, 2008. 